\title{
Polydimethylsiloxane and Castor Oil Comodified Waterborne Polyurethane
}

\author{
Xuan Ji, Yanming Zhou, Baoling Zhang, Caiying Hou, and Guozhang Ma
}

Shanxi Institute of Applied Chemistry, Taiyuan, Shanxi 030027, China

Correspondence should be addressed to Guozhang Ma; maguozhang@hotmail.com

Received 18 October 2013; Accepted 11 November 2013

Academic Editors: S. Fakirov and J. Puiggali

Copyright (C) 2013 Xuan Ji et al. This is an open access article distributed under the Creative Commons Attribution License, which permits unrestricted use, distribution, and reproduction in any medium, provided the original work is properly cited.

\begin{abstract}
In view of both improving properties of waterborne polyurethane (WPU) and sufficient utilization of renewable resources, a series of polydimethylsiloxane (PDMS) and castor oil (C.O.) comodified anionic WPUs with internal cross-linking was prepared through a prepolymer mixing process. The chemical structure of synthesized polymers was characterized by Fourier transforms infrared spectroscopy (FT-IR). In comparison with traditional linear WPU synthesized from petroleum-based polyols, these novel WPU films exhibited superior properties in solvent and water resistance, thermal stability, and mechanical strength, which suggest promising applications of these new environmentally friendly materials, particularly in the area of decorative and protective coatings. In addition, the results showed that with the increase in PDMS content in these co-modified WPUs the average particle size, the water resistance, and the thermal stability increased accordingly while the solvent resistance and the mechanical properties decreased.
\end{abstract}

\section{Introduction}

Waterborne polyurethane (WPU) dispersions, one of the most rapidly developing and active branches of PU chemistry and technology, have gained great attention in many commercial fields due to their superior properties and environmental advantages relative to conventional solvent-borne polyurethanes [1, 2]. However, some properties of WPU still need to be improved further, such as water and solvent resistance, thermal stability, and mechanical properties. The improvements of these properties have been achieved by copolymerizing or grafting of other polymers, external and internal cross-linking, blending or interpenetrating, polymer network formations, and modifying with nanoparticles [3-7].

Polydimethylsiloxane (PDMS) has many applications due to its unique properties, which arise mainly from its natural structure composed of inorganic $\mathrm{Si}-\mathrm{O}$ bond and organic graft methyl group. These interesting properties include low surface energy, biocompatibility, high thermal and oxidative stability, good water resistance, chemical inactivity, insulating stability, low glass transition temperature, and great molecular flexibility [8]. Combining the advantages of PDMS with those of WPU has attracted the attention of many researchers for a long time and PDMS has been introduced to WPU through both blending and copolymerization methods [816]. Although these linear PDMS-WPU "hybrids" showed much better heat and water resistance than that of WPU, the incorporation of PDMS often has a negative effect on the physical mechanical properties of WPU, because PDMS demonstrates poor mechanical property and abrasion resistance property. With the purpose to develop WPUPDMS copolymer with good mechanical strength and abrasion characteristics, cross-linking agents have been added to form the cross-linking structure during or shortly after film formation, also known as post-cross-linking or external cross-linking. For example, Zhang et al. synthesized a reactive PDMS-modified WPU by introducing a novel PDMS bearing carbonyl group at the side chain into the soft segment of WPU. After postadded dihydrazide, self-cross-linking was achieved by the reaction between the dihydrazide and carbonyl group during film formation. The film properties of this cross-linked PDMS-modified WPU showed improved water resistance and mechanical strength compared with that of WPU without postcross-linking [17]. However, there are few studies reporting introducing cross-linking internally by forming cross-linked prepolymer [18]. 
TABLE 1: Compositions of anionic WPU.

\begin{tabular}{|c|c|c|c|c|c|c|c|}
\hline \multirow{2}{*}{ Sample } & \multicolumn{7}{|c|}{ Composition } \\
\hline & PDMS & C.O. & PTMG & $\mathrm{IPDI}^{\mathrm{a}}$ & DMPA $^{b}$ & $\mathrm{TEA}^{\mathrm{c}}$ & $\mathrm{EDA}^{\mathrm{d}}$ \\
\hline WPU & 0 & 0 & 100 & 53.85 & 9.82 & 7.03 & 2.49 \\
\hline WPU-C & 0 & 20 & 80 & 57.14 & 10.03 & 7.18 & 2.66 \\
\hline WPU-P & 20 & 0 & 80 & 53.21 & 9.78 & 7.00 & 2.46 \\
\hline WPU-CP1 & 2 & 20 & 78 & 57.14 & 10.03 & 7.18 & 2.66 \\
\hline WPU-CP2 & 5 & 20 & 75 & 57.02 & 10.02 & 7.17 & 2.65 \\
\hline WPU-CP3 & 10 & 20 & 70 & 56.82 & 10.01 & 7.17 & 2.64 \\
\hline WPU-CP4 & 15 & 20 & 65 & 56.68 & 10.00 & 7.16 & 2.63 \\
\hline WPU-CP5 & 20 & 20 & 60 & 56.51 & 9.99 & 7.15 & 2.62 \\
\hline
\end{tabular}

${ }^{\mathrm{a}}$ Molar ratio of NCO/OH: $1.4 .{ }^{\mathrm{b}}$ Based on the weight of WPU polymer: $6 \% .{ }^{\mathrm{c}}$ Degree of neutralization: $95 \% .{ }^{\mathrm{d}}$ Degree of chain extension: $60 \%$.

On the other hand, polyurethanes derived from vegetable oils other than fossil feedstocks are put in the spotlight in an age of increasing oil prices, global warming, and other environmental concerns [19-21]. Castor oil (C.O.), among many kinds of vegetable oils, possesses many advantages like availability at low cost and average hydroxyl functionality of 2.7, making it suitable for use in isocyanate reactions to form cross-linked polyurethane while other vegetable oils usually need chemical modification of the triglyceride to produce polyols for polyurethane synthesis and long nonpolar fatty acid chains, rendering its formed film excellent properties such as hydrophobicity, softness, and flexing resistance [22]. Thus, C.O. is an important raw material in polyurethane manufacturing with applications in paints, coatings, inks, lubricants, and a wide variety of other fields. Compared with C.O.-based solvent-borne polyurethanes, however, limited attention has been paid to the synthesis of environmentally friendly WPU using C.O. as a renewable resource. Recently, Madbouly et al. have synthesized C.O.-based WPUs and systematically investigated the rheological behavior of these aqueous dispersions as a function of angular frequency, solid content, and temperature. The results provided fundamental insights into the relationships between polymer structure, rheological properties, and coating performance under typical conditions WPUs encounter during use [23]. Lu et al. also synthesized WPUs from C.O. and blended it with thermoplastic starch to obtain a novel biodegradable plastic with improved physical properties [24]. Since these WPUs used C.O. as the sole soft segment, the high functionality of C.O. may lead to gelation and higher cross-linking and therefore present difficulties in dispersing the resulting highly cross-linked PU prepolymer into water-this requires a large amount of solvent to reduce the viscosity of prepolymer and could hardly produce dispersions with high solid content. Besides, Qu and Chen synthesized both C.O.-based WPUs and C.O./poly(propylene glycol) mixing soft-segment WPUs and investigated their coating properties in order to replace single composition solvent-borne polyurethane coatings [25]. They found the film-forming property and elongation at break of the films of C.O.-based WPUs were undesirable when compared with those of C.O./poly(propylene glycol) mixing soft-segment WPUs.

In order to improve the key properties of WPU for use as coatings, adhesives, and related end use and achieve sufficient utilization of renewable resources, in this work, a series of PDMS and C.O. comodified anionic WPUs was prepared through a prepolymer mixing process, using isophorone diisocyanate (IPDI) as hard segment, PDMS and polytetramethylene glycol (PTMG) as soft segment, C.O. as cross-linking agent, dimethylol propionic acid (DMPA) as ion center, and diamine ethylene (EDA) as chain extender. By forming internally cross-linked WPUs, the prepared dispersions eliminate the necessity of postadd cross-linking agent, which usually causes the inconvenience in processing and application because of short pot-life. The effects of C.O. and the content of PDMS on both properties of dispersions and cast films were studied with focuses on particle size, water and solvent resistance, and thermal and mechanical properties.

\section{Experimental}

2.1. Materials. PTMG $\left(M_{n}=1000\right.$; Bayer, Germany), hydroxyl terminated PDMS $\left(M_{n}=1133\right.$; Shandong Dayi Chemicals, China), and C.O. $\left(M_{n}=932\right.$, average hydroxyl functionality of 2.7; Tianjin Tianda Chemicals, China) were vacuum-dried at $100^{\circ} \mathrm{C}$ for $2 \mathrm{~h}$ before use. IPDI (Bayer, Germany), DMPA (reagent grade; Tianjin Fuyu Chemicals, China), and dibutyltin dilaurate (DBTDL; Bayer, Germany) were used as received without further purification. Other solvents (reagent grade) used in synthesis and titration were purchased from Tianjin Fuyu Chemicals, China, and were immersed in $4 \AA$ molecular sieves for more than 1 week before use. Double-distilled and deionized water was used throughout.

2.2. Synthesis. The anionic WPU was synthesized by a prepolymer mixing process [2]. Basic formulations are given in Table 1. PTMG, hydroxyl terminated PDMS, and C.O. were charged into a $1000 \mathrm{~mL}$ four-necked flask equipped with mechanical stirrer, nitrogen inlet, condenser, and thermometer. While stirring, the mixture was kept at $80^{\circ} \mathrm{C}$ for $30 \mathrm{~min}$, followed by adding the catalyst DBDTL $(0.03 \mathrm{wt} \%$ based on the total solid) and IPDI to the homogenized mixtures. The reaction was carried out for $1 \mathrm{~h}$ at $80^{\circ} \mathrm{C}$ under dry nitrogen atmosphere. After that, DMPA dissolved in DMF $(w / w=1 / 2)$ was added to the flask. The reaction mixture 


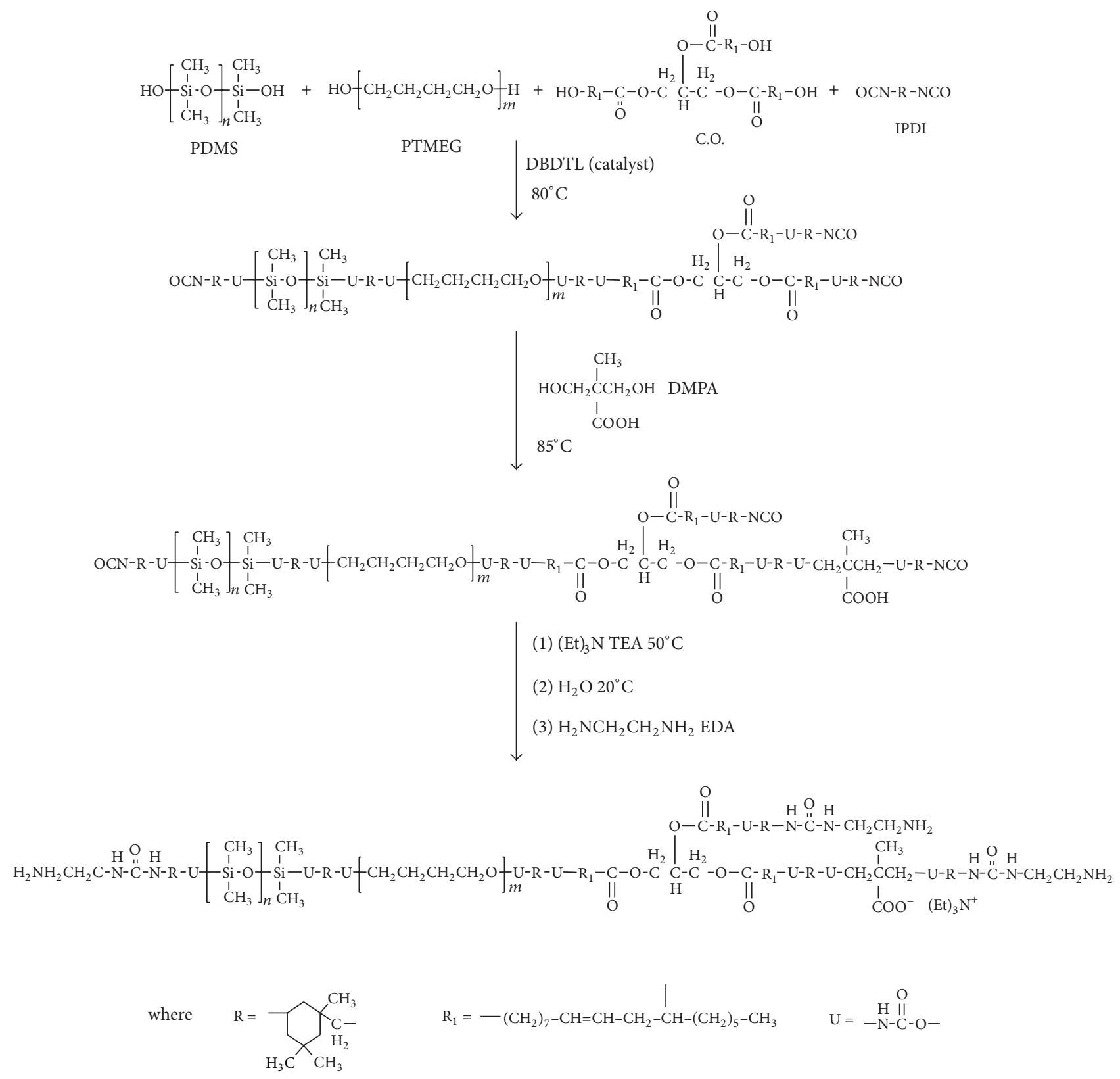

FIGURE 1: Synthesis of waterborne polyurethane.

was heated to $85^{\circ} \mathrm{C}$ until the NCO group content reached the theoretical value, which was determined by the standard dibutylamine back-titration method (ASTM D 1638). When the prepolymer was cooled to $50^{\circ} \mathrm{C}$, acetone was added to reduce the viscosity of the prepolymer followed by adding TEA to neutralize the carboxylic group in the side chain of prepolymer. After $30 \mathrm{~min}$ of neutralization, distilled water (70 wt\%) containing EDA was added to the reaction mixture with vigorous stirring (ca. $1000 \mathrm{rpm}$ ). The chain-extension reaction was allowed to proceed at $30^{\circ} \mathrm{C}$ until the NCO peak at $2270 \mathrm{~cm}^{-1}$ in the IR spectra had completely disappeared. After removal of acetone, the final WPU dispersion with 30\% solid content was obtained. The reaction scheme is shown in Figure 1.

2.3. Preparation of Films. The WPU dispersions were cast on leveled glass plates to allow them to dry at room temperature for 5 days and then at $60^{\circ} \mathrm{C}$ for $8 \mathrm{~h}$. After demoulding, the films were kept into a desiccator ready for testing.

\section{Characterization and Measurements}

FT-IR spectra were recorded on a Nicolet 360 Fourier Transform Infrared Spectrometer (USA) with KBr pellets. For each 
sample, 32 scans at a resolution of $2 \mathrm{~cm}^{-1}$ were collected in the transmittance mode in the wavenumber range of 4000$400 \mathrm{~cm}^{-1}$.

Average particle sizes were determined by the dynamic light scattering method with Bettersize 2000 Intelligent Laser Particle Size Analyzer (Dandong Bettersize Instruments Ltd., China) at $25^{\circ} \mathrm{C}$ and the measured wavelength was $635 \mathrm{~nm}$. The aqueous WPU dispersions, diluted in distilled deionized water, were submitted to ultrasound in the equipment before analysis.

The viscosity of WPU dispersion was determined by NDJ79 Rotational Viscometer (Shanghai Ande Instruments Ltd., China) at $25^{\circ} \mathrm{C}$.

The soluble fraction (SF) of the WPU films in toluene was determined as follows: samples of about $0.5 \mathrm{~g}$ were cut from the WPU films, weighed, and immersed in an excess of toluene for $24 \mathrm{~h}$ at room temperature under stirring. Then, the samples were removed from the solvent and dried under a vacuum at $80^{\circ} \mathrm{C}$ for $24 \mathrm{~h}$. The dried samples were then weighed, and the SF was calculated by the following equation:

$$
\mathrm{SF}(\%)=\frac{W_{i}-W_{e}}{W_{i}} \times 100 \%,
$$

where $W_{i}$ and $W_{e}$ are the weight of the initial and the extracted WPU films, respectively.

Water absorption (WA) of the WPU films was tested as follows: a piece of the WPU film with known weight $\left(m_{1}\right)$ was immersed in distilled water at room temperature; after $72 \mathrm{~h}$ of equilibration the sample was taken out. Excess water on film surface was wiped up using filter paper and the film weighed again $\left(m_{2}\right)$. The WA of the film was calculated as follows:

$$
\mathrm{WA}(\%)=\frac{m_{2}-m_{1}}{m_{1}} \times 100 \%
$$

The contact angle measurements were performed with a KRUSS EasyDrop apparatus (Germany). A water droplet was deposited on the sample film surface and the droplet shape was recorded. A video camera and image analysis software (EasyDrop Contact Angle Measuring System) were used to determine the contact angle evolution. Results are the average values of five measurements.

A HCT-1 thermogravimeter (Beijing Henven Instruments Ltd., China) was used to measure the weight loss of the WPU films under an air atmosphere. The samples were heated from 100 to $650^{\circ} \mathrm{C}$ at a heating rate of $20^{\circ} \mathrm{C} / \mathrm{min}$. Generally, $10-$ $15 \mathrm{mg}$ samples were used for the thermogravimetric analysis (TGA).

The Shore A hardness of the films was measured at room temperature by HT-6510A Shore A Hardness Tester (LandTek Instruments Ltd., China) according to ASTM D 2240. Results are the average values of five measurements.

The tensile strength and elongation at break of the films were determined by Electronic Universal Testing Machines (Shenzhen SANs Testing Machine Co. Ltd., China) at room temperature with a crosshead speed of $100 \mathrm{~mm} / \mathrm{min}$. Dumbbell specimens were cut from WPU films of $0.4-0.8 \mathrm{~mm}$ thickness by a sheet-punching machine. An average value of five replicates of each sample was taken.

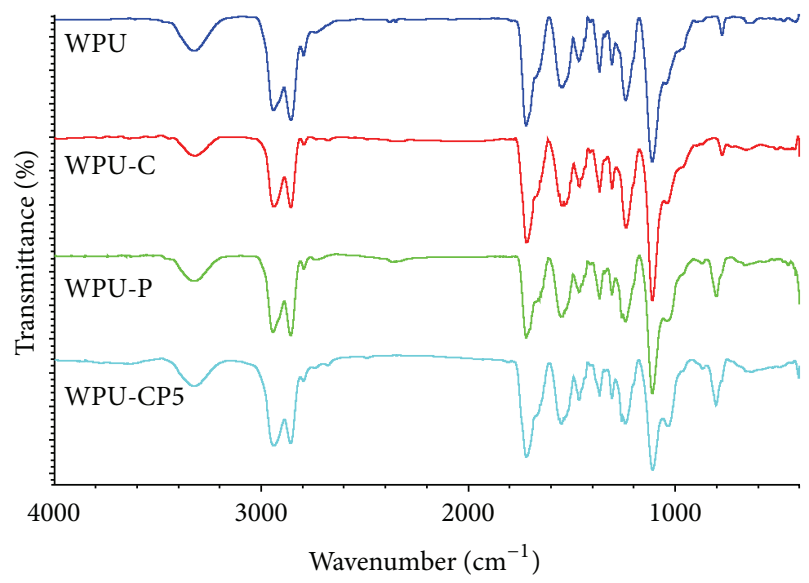

FIGURE 2: FT-IR spectra of the WPU samples.

\section{Results and Discussion}

4.1. Characterization by FT-IR. The FT-IR spectra of the representative WPU samples in this study are shown in Figure 2. The absence of band at $2250-2270 \mathrm{~cm}^{-1}$ in all samples confirmed the absence of a free NCO group in the polymer structure, which indicated the completion of the reaction.

For sample WPU, the most important characteristic features are the presence of peaks at $3322 \mathrm{~cm}^{-1}(\mathrm{~N}-\mathrm{H}$ stretching vibration from urethane group), 2942 and $2856 \mathrm{~cm}^{-1}\left(\mathrm{CH}_{2}\right.$ symmetric and asymmetric stretching vibrations), $1718 \mathrm{~cm}^{-1}$ ( $\mathrm{C}=\mathrm{O}$ stretching vibration from urethane group), $1541 \mathrm{~cm}^{-1}$ (C-N stretching and $\mathrm{N}-\mathrm{H}$ bending vibrations from urethane group), 1465 and $1365 \mathrm{~cm}^{-1}$ (C-H asymmetric and symmetric bending vibrations from methylene group), and $1238 \mathrm{~cm}^{-1}$ (C-O-C asymmetric stretching vibration). These peaks confirmed the formation of the desired WPU structure. For sample WPU-C, compared with sample WPU, the main characteristic peaks are the same except the $\mathrm{C}=\mathrm{O}$ stretching vibration band of the ester group in C.O. which was shifted from $1750-1744 \mathrm{~cm}^{-1}$ to $1720 \mathrm{~cm}^{-1}$ because of the formation of hydrogen bonding. For sample WPU-P, compared with sample WPU, the presence of peaks at $1261 \mathrm{~cm}^{-1}$ (C-H symmetric bending vibration in $\left.\mathrm{Si}-\mathrm{CH}_{3}\right), 1020 \mathrm{~cm}^{-1}$ ( $\mathrm{Si}-\mathrm{O}-\mathrm{Si}$ stretching vibration), and $800 \mathrm{~cm}^{-1}$ (C-H rocking vibration in $\mathrm{Si}-\mathrm{CH}_{3}$ ) confirmed the incorporation of a PDMS segment in WPU structure.

For sample WPU-CP5, the presence of characteristic peaks of urethane, C.O., and PDMS as shown in samples of WPU, WPU-C, and WPU-P confirmed the formation of the designed PU structure. Hydrogen bonding is a very important feature in polyurethanes and has significant effect on the material properties. FT-IR spectroscopy as an effective tool has been used to investigate the hydrogen bonding of polyurethanes [19]. In sample WPU, the $\mathrm{C}=\mathrm{O}$ stretching vibration peak appeared at $1718 \mathrm{~cm}^{-1}$ and the $\mathrm{N}-\mathrm{H}$ stretching vibration peak at $3322 \mathrm{~cm}^{-1}$, while in sample WPU-CP5, the $\mathrm{C}=\mathrm{O}$ stretching vibration peak appeared at $1719 \mathrm{~cm}^{-1}$ 

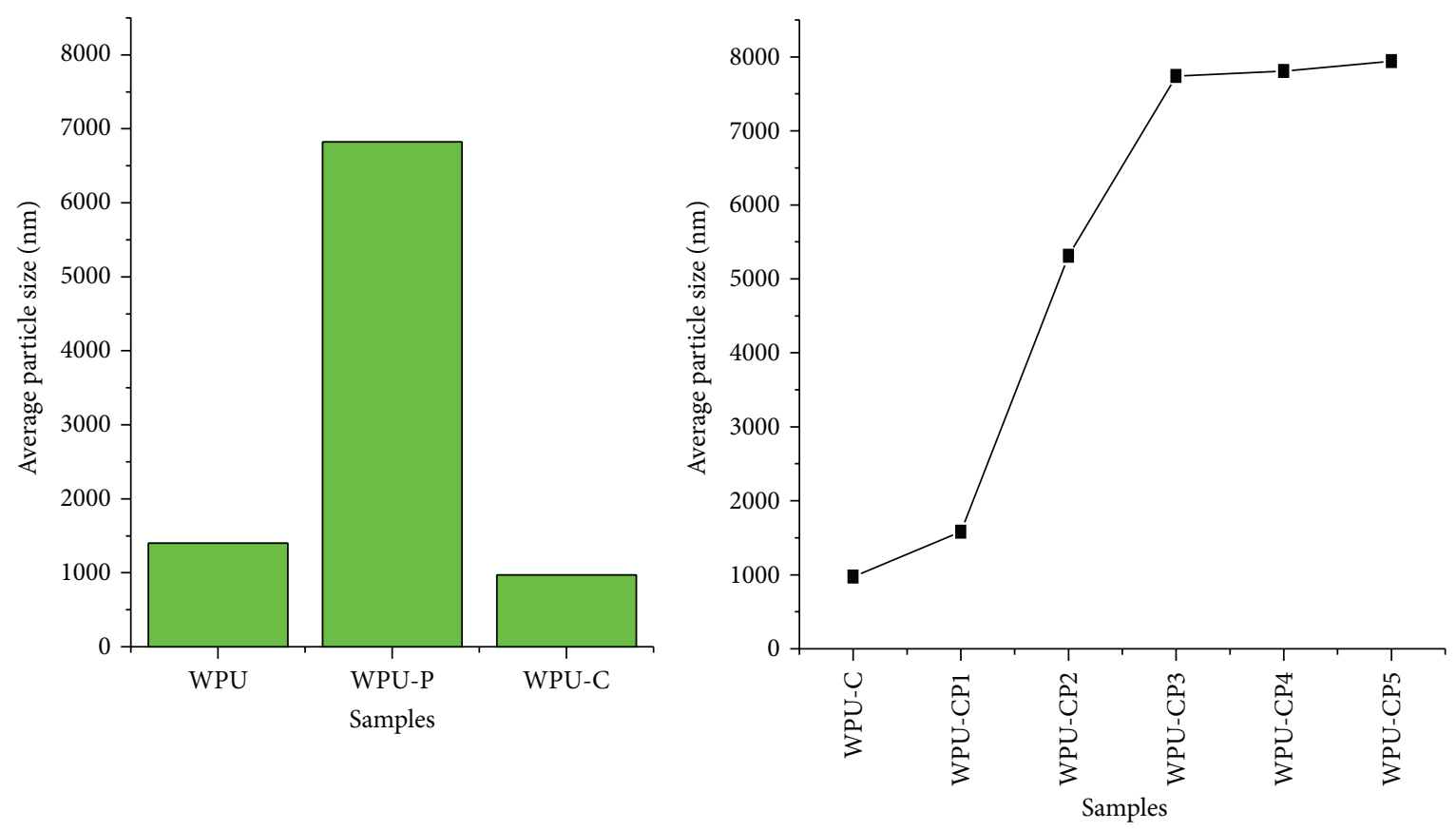

FIGURE 3: Average particle size of WPU dispersion.

and the $\mathrm{N}-\mathrm{H}$ stretching vibration peak at $3325 \mathrm{~cm}^{-1}$, both peaks shifting to higher wavenumbers, which indicate that less hydrogen bonds have been formed for sample WPUCP5 than for sample WPU. Consequently, there is a decreased intermolecular interaction of the hard segments with the soft segments for sample WPU-CP5. This is because the incorporation of PDMS in PU structure widened the separation extent of the soft and hard phases for the incompatibility between PDMS and urethanes.

4.2. Properties of WPU Dispersion. In WPU dispersions, the average particle size is important for its stability and application [26]. For example, relatively large particles are preferred in many surface coatings to facilitate rapid drying, and relatively small particles are desirable when deep penetration of the dispersion into substrate is an essential step. Figure 3 shows the average particle size for each WPU sample in this study.

For samples WPU-C, WPU-CP1, WPU-CP2, WPU-CP3, WPU-CP4, and WPU-CP5, it was found that the average particle size increased from $972 \mathrm{~nm}$ to $7941 \mathrm{~nm}$ as the PDMS content in the soft segment of WPU increased from $0 \%$ to $20 \%$. In the process of dispersion forming, the average particle size of droplet (Dp) is well described by the following equation:

$$
\frac{\mathrm{Dp}}{D}=0.08(1+0.47 \emptyset) \mathrm{We}^{-0.6},
$$

where $D$ is the diameter of stirrer, $\emptyset$ is phase volume of the dispersed phase, and We is Webers' criteria, defined as:

$$
\mathrm{We}=\frac{N^{2} D^{3} \rho}{\sigma},
$$

where $N$ is rotating speed of stirrer, $\rho$ is mass density of continuous phase, and $\sigma$ is interfacial tension [14]. In this study, the above-mentioned process parameters (including dispersing temperature) for each sample were constant. Other factors that would influence the average particle size such as the $\mathrm{NCO} / \mathrm{OH}$ ratio of composition, degree of neutralization, and, particularly, ionic content were designed similarly [27, 28]. Therefore, according to the equation the asymptotic increase of the average particle size is attributed to the increase of the interfacial tension, which was caused by the increased concentration of hydrophobic PDMS in particles. This also explains why a sample modified by PDMS (WPU$\mathrm{P})$ showed much larger average particle size than that of those nonmodified by PDMS (WPU and WPU-C).

All WPU dispersions in this study showed low viscosity (see Table 2, range from 10 to $25 \mathrm{Mpa} \cdot \mathrm{s}$ ), which indicates that the distances among particles are relatively large due to effective emulsification, so the dispersions mainly showed the flowing characteristic of solvent (water) under shear force. The viscosity of dispersions modified with C.O. was slightly higher than that of non-modified ones and this could be attributed to the higher cross-linking density and more hydrophobicity rendered by the C.O. segment.

4.3. Solvent and Water Resistance Properties of the WPU Films. Figure 4 shows the SF of the WPU films. It was found that the SF of C.O. modified WPU samples (2-3.5\% for WPUC, WPU-CP1, WPU-CP2, WPU-CP3, WPU-CP4, and WPUCP5) was much lower than that of nonmodified ones $(>7 \%$ for WPU and WPU-P). This indicates that C.O. indeed functioned as an effective internal cross-linking agent for WPU, and the effects of C.O. on improvements on film solvent resistance are of great interest because WPUs are 
TABLE 2: Viscosity of the dispersions and the thermal and mechanical properties of the films.

\begin{tabular}{|c|c|c|c|c|c|c|c|}
\hline \multirow{2}{*}{ Sample } & \multirow{2}{*}{$\begin{array}{l}\text { Viscosity } \\
\text { Mpa·s }\end{array}$} & \multicolumn{3}{|c|}{ TGA $\left({ }^{\circ} \mathrm{C}\right)$} & \multirow{2}{*}{$\begin{array}{l}\text { Tensile strength } \\
(\mathrm{MPa})\end{array}$} & \multirow{2}{*}{$\begin{array}{c}\text { Elongation at } \\
\text { break (\%) }\end{array}$} & \multirow{2}{*}{$\begin{array}{l}\text { Hardness } \\
\text { (Shore A) }\end{array}$} \\
\hline & & $T_{5}$ & $T_{10}$ & $T_{50}$ & & & \\
\hline WPU & 12 & 268.4 & 291.4 & 390.8 & 9.38 & 801 & 72 \\
\hline WPU-C & 18 & 267.0 & 285.1 & 395.3 & 17.23 & 615 & 82 \\
\hline WPU-P & 10 & 256.9 & 276.3 & 384.0 & 9.15 & 631 & 68 \\
\hline WPU-CP1 & 18 & 271.0 & 294.9 & 389.6 & 14.65 & 334 & 70 \\
\hline WPU-CP2 & 19 & 259.7 & 281.1 & 395.8 & 12.46 & 305 & 67 \\
\hline WPU-CP3 & 17 & 262.0 & 283.1 & 396.0 & 11.06 & 279 & 62 \\
\hline WPU-CP4 & 21 & 263.7 & 283.3 & 396.6 & 10 & 256 & 59 \\
\hline WPU-CP5 & 19 & 269.0 & 292.9 & 397.6 & 13.4 & 348 & 51 \\
\hline
\end{tabular}
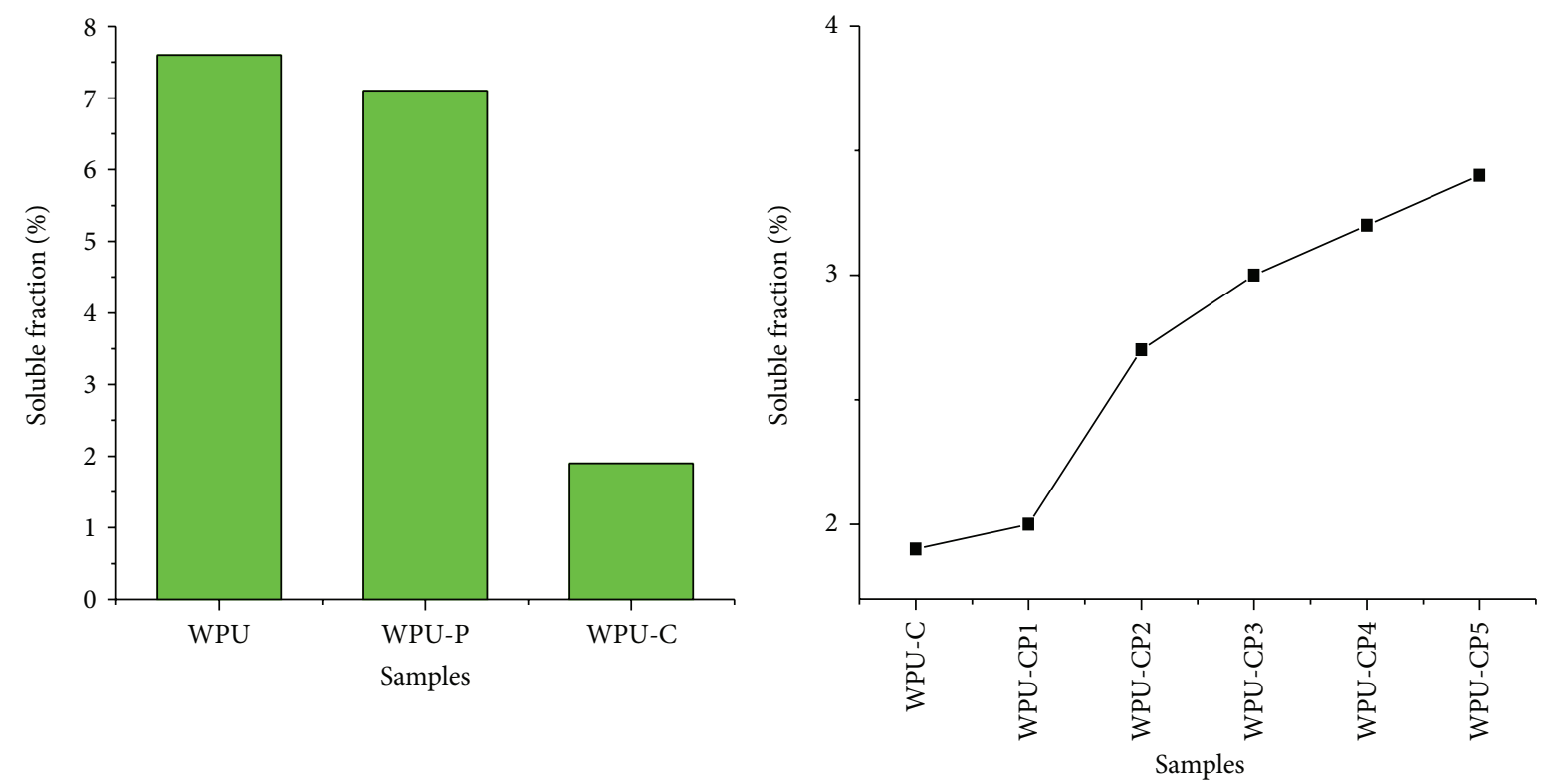

FIGURE 4: Soluble Fraction of the WPU films.

often used as special coatings, where solvent resistance is a critical property for their end application performance [3]. Besides, from WPU-CP1 to WPU-CP5 series it was found that the SF becomes higher as the PDMS content increases, which is probably caused by more small soluble molecules generated as hydroxyl terminated PDMS which may have some termination effects.

The water resistance of WPU film is a critical property and has significant influence on many applications. However, the water resistance property of WPU film is always interior to the solvent-borne two-component polyurethanes, which relates to the very nature of WPU (ionic groups in WPU ionomers are hydrophilic) [2]. WA and water contact angle of WPU film are two effective methods to measure the water resistance property $[15,18]$.

Figures 5 and 6 show the WA and water contact angle for the WPU films in this study, respectively. Figure 7 shows the water contact angle image of representative samples. For samples WPU-C, WPU-CP1, WPU-CP2, WPU-CP3, WPUCP4, and WPU-CP5, it was found that as the content of PDMS in the soft segment of WPU increased from $0 \%$ to
$20 \%$ the WA of the WPU films decreased from $20.3 \%$ to $6 \%$, whereas the water contact angle increased from $54^{\circ}$ to $100^{\circ}$. This should be attributed to the hydrophobicity of the PDMS molecules. During film formation, the PDMS soft segment has migrated to the surface layer because its water affinity is much lower than that of WPU chain $[12,17]$. When PDMS was rich in the surface layer, the film could not be immerse in water because of strong hydrophobic $\mathrm{Si}-\mathrm{O}-\mathrm{Si}$ bonds in PDMS [10]. Therefore, as the content of PDMS increased, the concentration of PDMS in surface layer of WPU film increased accordingly, and this led to the decrease of WA and the increase of water contact angle.

From the above results it is shown that the C.O. and PDMS co-modified WPU samples in this study have advantages in both solvent and water resistance properties when compared with non-modified ones or only modified by C.O. or PDMS ones.

4.4. Thermal Properties of the WPU Films. The thermal property of the WPU films was studied by TGA. Typical TGA curves for the WPU films are shown in Figure 8, and the TGA 

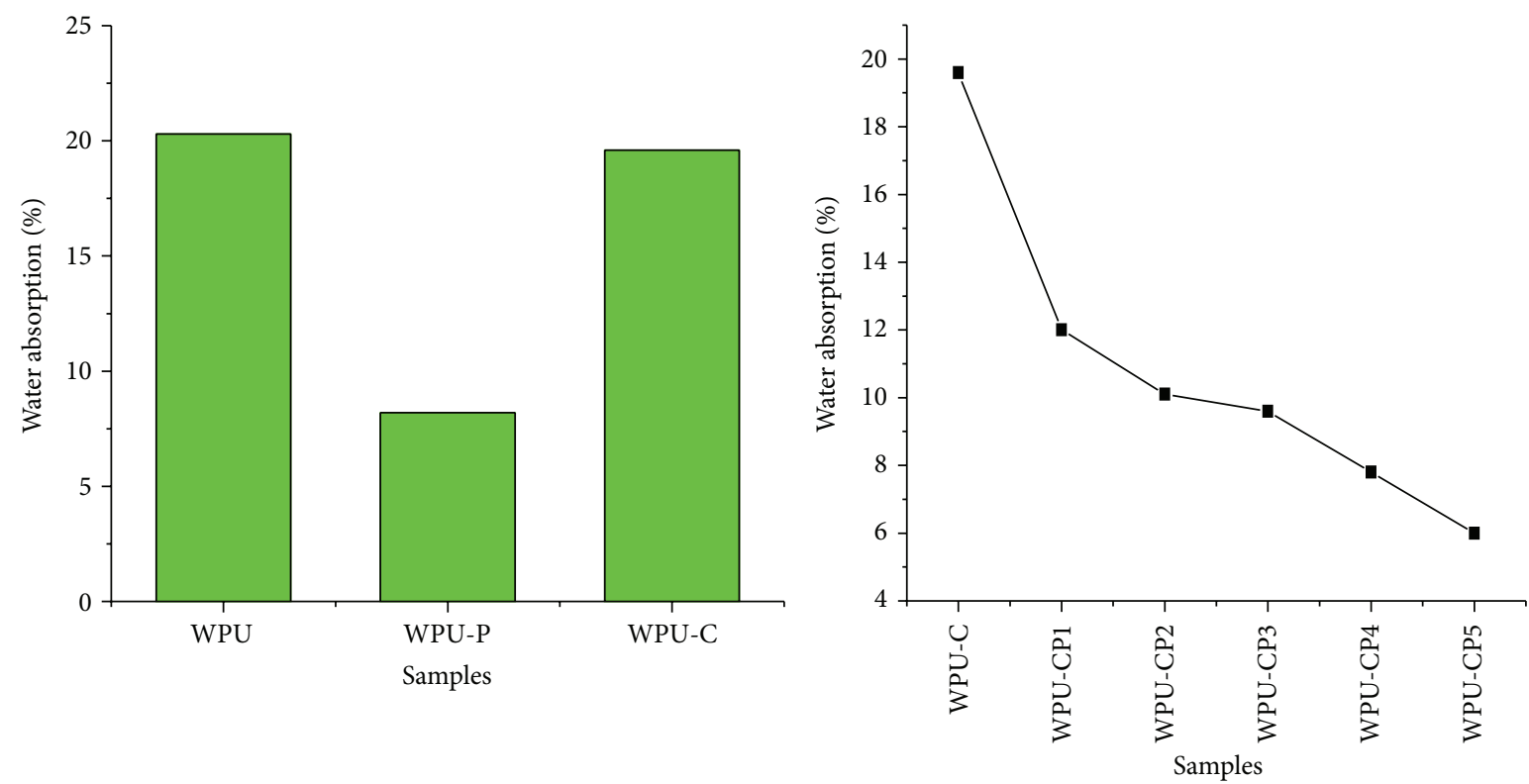

FIgURE 5: Water absorption of the WPU films.
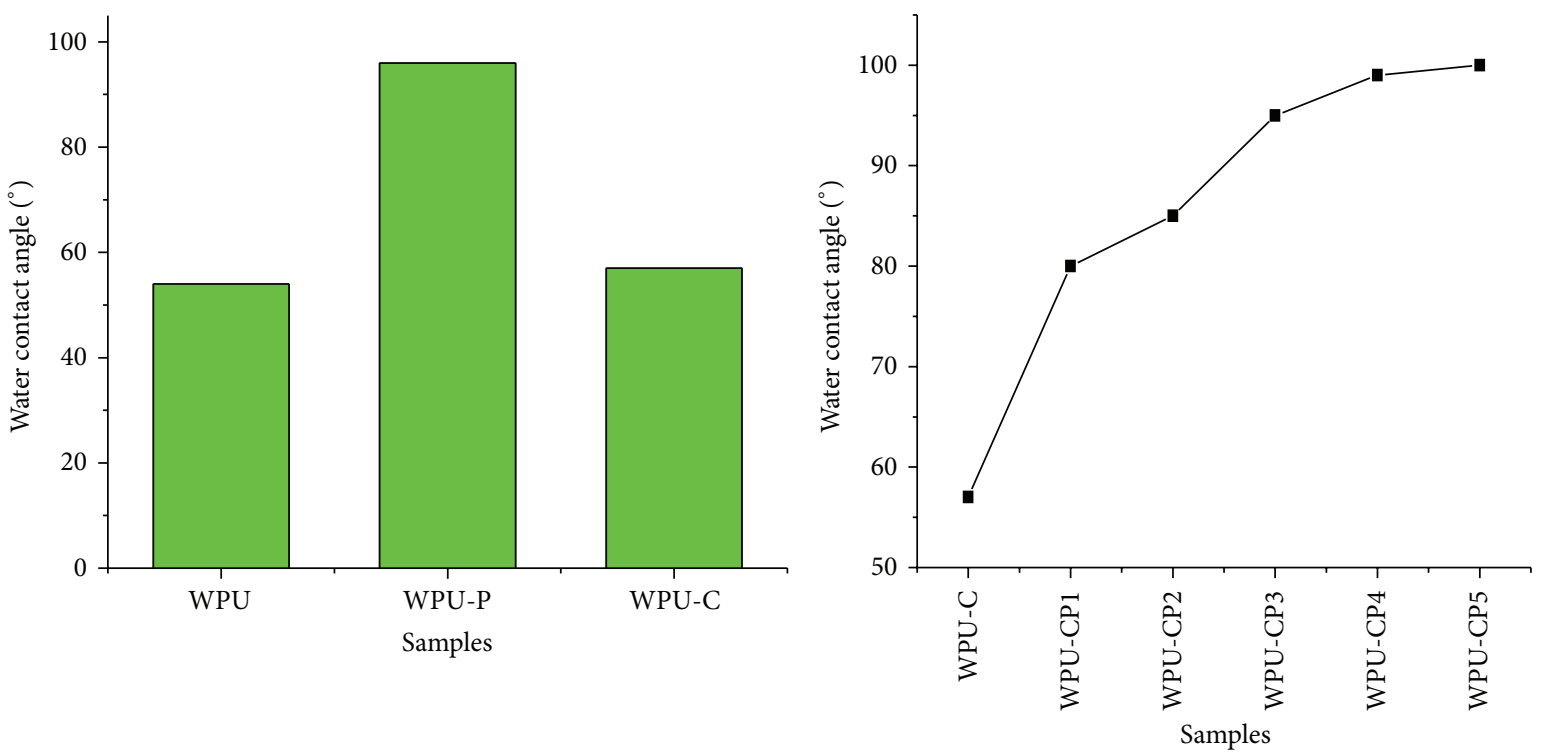

FIgURE 6: Water contact angle of WPU films.

data are summarized in Table 2. Generally, polyurethanes exhibit relatively low thermal stability because of the presence of labile urethane groups and the degradation mechanism is very complex due to the variety of products formed in the process [19]. The onset of urethane bond dissociation is somewhere $<300^{\circ} \mathrm{C}$, depending upon the type of isocyanate and polyol employed. In the present study, the WPU films undergo more than one thermal degradation process. The degradation of the WPU films that is observed in the range of $150-300^{\circ} \mathrm{C}$ can be attributed to decomposition of the urethane and urea bonds, which takes place through the dissociation between isocyanate and alcohol, the formation of primary amines and olefins, or the formation of secondary amines, which results in the loss of carbon dioxide from the urethane bond. The degradation process in the temperature range of $300-400^{\circ} \mathrm{C}$ is attributed to the degradation of PTMG soft segment and C.O. chain scission. The range of $400-530^{\circ} \mathrm{C}$ is mainly ascribed to the degradation of PDMS soft segment.

For samples WPU, WPU-CP1, WPU-CP2, WPU-CP3, WPU-CP4, and WPU-CP5, it was found that as the PDMS content increased, the 50\% degradation temperature $\left(T_{50}\right)$ increased from $390.8^{\circ} \mathrm{C}$ to $397.6^{\circ} \mathrm{C}$. This increase in degradation temperature suggests that the role of PDMS is to prevent the degradation of polyurethane backbone. Feasibility and facility of rotation along the $\mathrm{Si}-\mathrm{O}$ linkage can compel the polysiloxane molecule to absorb a part of heat energy 


\section{I}

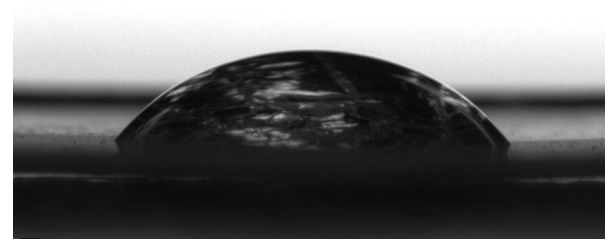

Sample WPU

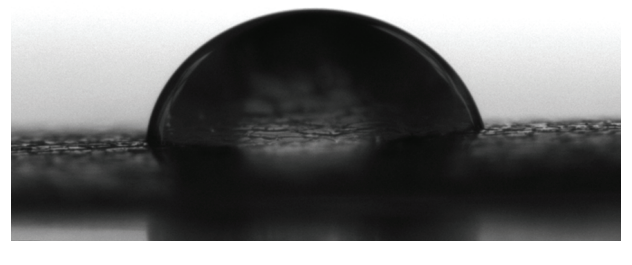

Sample WPU-CP1

FIGURE 7: Water contact angle image of representative WPU samples.

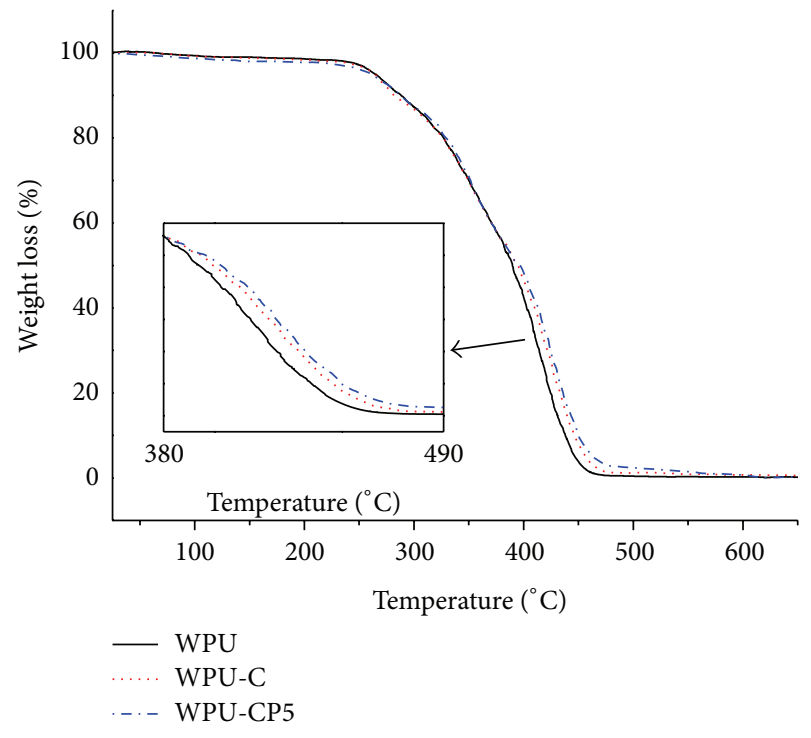

FIgURE 8: TGA curves for the typical WPU films.

thereby raising the requirement of temperature. For samples WPU and WPU-C, all degradation temperatures of the C.O. modified sample WPU-C were found to be higher than those of linear sample WPU. This is because WPU-C has higher cross-linking density rendered by C.O. and it gives more compact structure and more secondary interaction than the linear one, whereas when comparing sample WPU-P with WPU, $T_{5}, T_{10}$, and $T_{50}$ all began to decrease, which could be attributed to enhanced microphase separation between PDMS and polyurethane when the content of PDMS in WPUs was too much.

From the previous analyses and the curve profile in Figure 8, it was found that either PDMS or C.O. could improve the thermal stability of WPU, and PDMS and C.O. co-modified WPUs showed synergetic improvements in the thermal stability to some degree.

4.5. Mechanical Properties of the WPU Films. Table 2 presents the physical properties of WPU films. It is well known that the physical properties of WPU films depend on the molecular structure of raw materials and the molar ratio of $\mathrm{NCO} / \mathrm{OH}$ [29]. Since the molar ratio of $\mathrm{NCO} / \mathrm{OH}$ is 1.4 in all samples in this study, the type and proportion of raw materials would be the main influencing factors.

Comparing sample WPU-C with sample WPU, the hardness and tensile strength were both increased while elongation at break decreased, from which it was clearly seen that cross-linking was indeed enhanced in the C.O. modified WPU. For samples WPU-C, WPU-CP1, WPUCP2, WPU-CP3, WPU-CP4, and WPU-CP5, the Shore A hardness decreased with increased PDMS content-on the one hand, this is because the soft PDMS segment tended to stay on the top of the films and this would reduce the hardness of the films; on the other hand, the intermolecular bonding between hard segments and soft segments (such as hydrogen bond) was reduced due to the higher phase separation caused by the higher PDMS content. As the content of PDMS increased (WPU-CP1 to WPU-CP4), the tensile strength and elongation at break decreased from $14.65 \mathrm{MPa}$ to $10 \mathrm{MPa}$ and from $335 \%$ to $256 \%$, respectively. These decreases could be attributed to the lower mechanical properties of the PDMS component. However, as the content of PDMS further increased (WPU-CP5), both the tensile strength and elongation at break were increased. This change is probably due to the formation of double-layered films with the upper thin layer mainly composing PDMS and lower layer mainly composing PU chains when the content of PDMS exceed the some level; thus the overall mechanical properties partly showed nonmodified WPU's characteristics (the lower layer). This mechanism could also explain the mechanical properties of sample WPU-P when compared with sample WPU. Generally speaking, with appropriate PDMS content, the PDMS and C.O. co-modified WPUs showed improved mechanical properties than non-modified WPUs.

\section{Conclusions}

A series of PDMS and C.O. co-modified anionic WPUs was synthesized by mixed soft-segment copolymerization. The chemical structure of synthesized polymers was confirmed by 
FT-IR spectroscopy. The increase in PDMS content in these co-modified WPUs increased the average particle size, and the water resistance, the thermal stability while decreasing the solvent resistance and the mechanical properties. Compared with traditional linear WPU from petroleum-based polyols, these novel WPU films combined the advantages of PDMS and C.O. with those of WPU and exhibited better properties in solvent and water resistance, thermal stability, and mechanical strength, which suggests promising applications of these new environmentally friendly PDMS and renewable plant oil co-modified PU materials, particularly in the area of decorative and protective coatings.

\section{Acknowledgments}

This study was supported by the Shanxi Scholarship Council of China (2012-8), and Scientific Research Foundation of Shanxi Province, China (no. 20111101059), and the Youth Natural Science Foundation of Shanxi Province, China, which are gratefully acknowledged.

\section{References}

[1] F. M. B. Coutinho, M. C. Delpech, and L. S. Alves, "Anionic waterborne polyurethane dispersions based on hydroxylterminated polybutadiene and poly(propylene glycol): synthesis and characterization," Journal of Applied Polymer Science, vol. 80, pp. 566-572, 2001.

[2] B. K. Kim, "Aqueous polyurethana dispersions," Colloid and Polymer Science, vol. 274, no. 7, pp. 599-611, 1996.

[3] X. Zhu, Q. Zhang, L. Liu, X. Z. Kong, and S. Feng, "Synthesis and characterization of a new compound bearing ketone and hydroxyl groups for preparation of ambient temperature selfcrosslinking waterborne polyurethanes," Progress in Organic Coatings, vol. 59, no. 4, pp. 324-330, 2007.

[4] P. Florian, K. K. Jena, S. Allauddin, R. Narayan, and K. V. S. N. Raju, "Preparation and characterization of waterborne hyperbranched polyurethane-urea and their hybrid coatings," Industrial and Engineering Chemistry Research, vol. 49, no. 10, pp. 4517-4527, 2010.

[5] S. Vlad, A. Vlad, and S. Oprea, "Interpenetrating polymer networks based on polyurethane and polysiloxane," European Polymer Journal, vol. 38, no. 4, pp. 829-835, 2002.

[6] G. Ma, W. Liu, X. Liu, J. Wu, T. Yan, and B. Xu, "Preparation and properties of polymerizable silica hybrid nanoparticles with tertiary amine structure," Progress in Organic Coatings, vol. 71, no. 1, pp. 83-88, 2011.

[7] Y. Chen, S. Zhou, H. Yang, and L. Wu, "Structure and properties of polyurethane/nanosilica composites," Journal of Applied Polymer Science, vol. 95, no. 5, pp. 1032-1039, 2005.

[8] J. Kozakiewicz, "Polysiloxaneurethanes: new polymers for potential coating applications," Progress in Organic Coatings, vol. 27, no. 1-4, pp. 123-131, 1996.

[9] M.-S. Yen and P.-Y. Tsai, "Effects of soft segments on the surface properties of polydimethylsiloxane waterborne polyurethane prepolymer blends and treated nylon fabrics," Journal of Applied Polymer Science, vol. 115, no. 6, pp. 3550-3558, 2010.

[10] M.-S. Yen, P.-Y. Tsai, and P.-D. Hong, "The solution properties and membrane properties of polydimethylsiloxane waterborne polyurethane blended with the waterborne polyurethanes of various kinds of soft segments," Colloids and Surfaces A, vol. 279, no. 1-3, pp. 1-9, 2006.

[11] M.-S. Yen and P.-Y. Tsai, "Effects on the structure and properties of membranes formed by blending polydimethylsiloxane polyurethane into different soft-segment waterborne polyurethanes," Journal of Applied Polymer Science, vol. 102, no. 1, pp. 210-221, 2006.

[12] M.-S. Yen and P.-Y. Tsai, "Study on polyethylene glycol/polydimethylsiloxane mixing soft-segment waterborne polyurethane from different mixing processes," Journal of Applied Polymer Science, vol. 90, no. 1, pp. 233-243, 2003.

[13] M. M. Rahman, A. Hasneen, H.-D. Kim, and W. K. Lee, "Preparation and properties of polydimethylsiloxane (PDMS)/ polytetramethyleneadipate glycol (PTAd)-based waterborne polyurethane adhesives: effect of PDMS molecular weight and content," Journal of Applied Polymer Science, vol. 125, no. 1, pp. 88-96, 2012.

[14] H. Wang, Y. Shen, G. Fei, X. Li, and Y. Liang, "Micromorphology and phase behavior of cationic polyurethane segmented copolymer modified with hydroxysilane," Journal of Colloid and Interface Science, vol. 324, no. 1-2, pp. 36-41, 2008.

[15] Y.-H. Lee, E.-J. Kim, and H.-D. Kim, "Synthesis and properties of waterborne poly(urethane urea)s containing polydimethylsiloxane," Journal of Applied Polymer Science, vol. 120, no. 1, pp. 212-219, 2011.

[16] G. Fei, Y. Shen, H. Wang, and Y. Shen, "Effects of polydimethylsiloxane concentration on properties of polyurethane/polydimethylsiloxane hybrid dispersions," Journal of Applied Polymer Science, vol. 102, no. 6, pp. 5538-5544, 2006.

[17] M. Zhang, Y. Wu, H. Wu, and Q. Zhang, "Study on reactive polydimethylsiloxane-modified waterborne polyurethanes," Journal of Polymer Research, vol. 19, 2011.

[18] X. Li, G. Fei, and H. Wang, "Mechanical and surface properties of membranes prepared from waterborne cationic hydroxylterminated polydimethylsiloxane/polyurethane surfactant-free micro-emulsion," Journal of Applied Polymer Science, vol. 100, no. 1, pp. 40-46, 2006.

[19] Y. Lu and R. C. Larock, "Soybean-oil-based waterborne polyurethane dispersions: effects of polyol functionality and hard segment content on properties," Biomacromolecules, vol. 9, no. 11, pp. 3332-3340, 2008.

[20] G. Lligadas, J. C. Ronda, M. Galiá, and V. Cádiz, "Plant oils as platform chemicals for polyurethane synthesis: current state-ofthe-art," Biomacromolecules, vol. 11, no. 11, pp. 2825-2835, 2010.

[21] Y.-C. Tu, P. Kiatsimkul, G. Suppes, and F.-H. Hsieh, "Physical properties of water-blown rigid polyurethane foams from vegetable oil-based polyols," Journal of Applied Polymer Science, vol. 105, no. 2, pp. 453-459, 2007.

[22] D. S. Ogunniyi, "Castor oil: a vital industrial raw material," Bioresource Technology, vol. 97, no. 9, pp. 1086-1091, 2006.

[23] S. A. Madbouly, Y. Xia, and M. R. Kessler, "Rheological behavior of environmentally friendly castor oil-based waterborne polyurethane dispersions," Macromolecules, vol. 46, pp. 4606-4616, 2013.

[24] Y. Lu, L. Tighzert, P. Dole, and D. Erre, "Preparation and properties of starch thermoplastics modified with waterborne polyurethane from renewable resources," Polymer, vol. 46, no. 23, pp. 9863-9870, 2005.

[25] J. Qu and H. Chen, "Studies on syntheses and properties of waterborne polyurethane resin from castor oil," Chemistry and Industry of Forest Products, vol. 24, pp. 78-82, 2004. 
[26] B. K. Kim and J. C. Lee, "Waterborne polyurethanes and their properties," Journal of Polymer Science A, vol. 34, no. 6, pp. 10951104, 1996.

[27] J. Yoon Jang, Y. Kuk Jhon, I. Woo Cheong, and J. Hyun Kim, "Effect of process variables on molecular weight and mechanical properties of water-based polyurethane dispersion," Colloids and Surfaces A, vol. 196, no. 2-3, pp. 135-143, 2002.

[28] S. M. Cakić, M. Špírková, I. S. Ristić, J. K. B.-Simendić, M. M.-Cincović, and R. Poręba, "The waterborne polyurethane dispersions based on polycarbonate diol: Effect of ionic content," Materials Chemistry and Physics, vol. 138, pp. 277-285, 2013.

[29] F. Levine, J. Escarsega, and J. La Scala, "Effect of isocyanate to hydroxyl index on the properties of clear polyurethane films," Progress in Organic Coatings, vol. 74, pp. 572-581, 2012. 

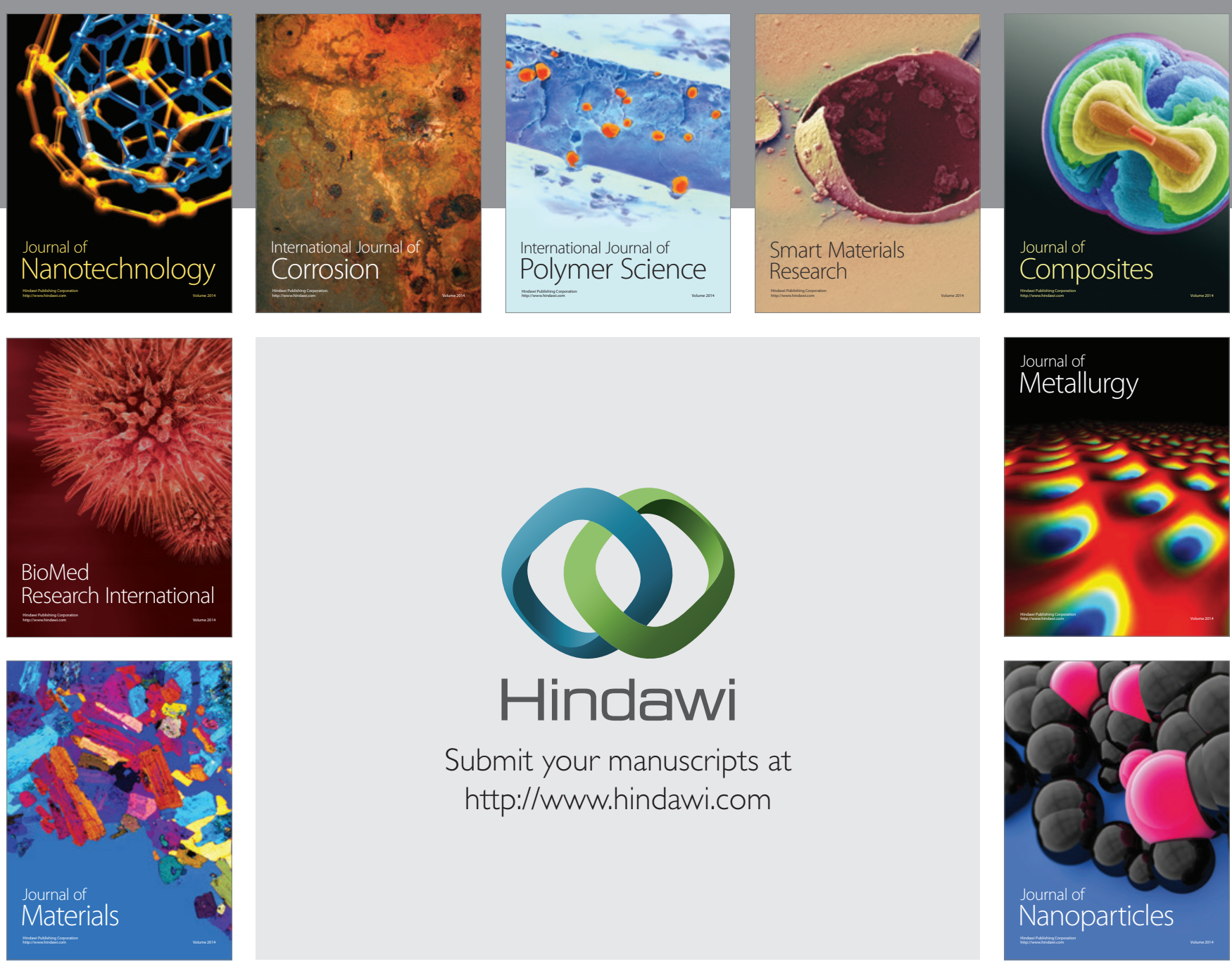

Submit your manuscripts at http://www.hindawi.com
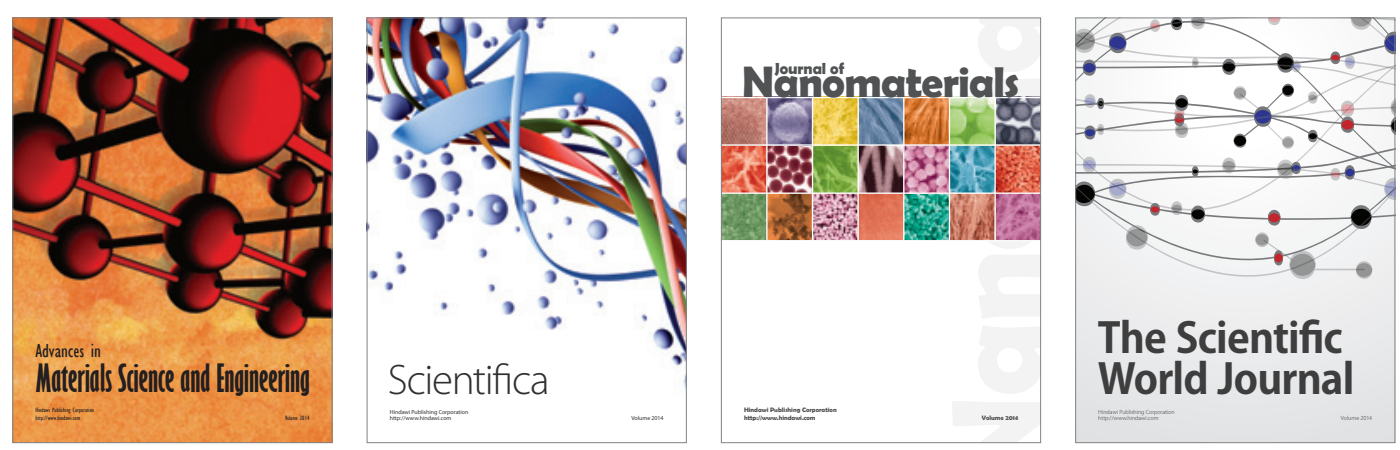

\section{The Scientific World Journal}
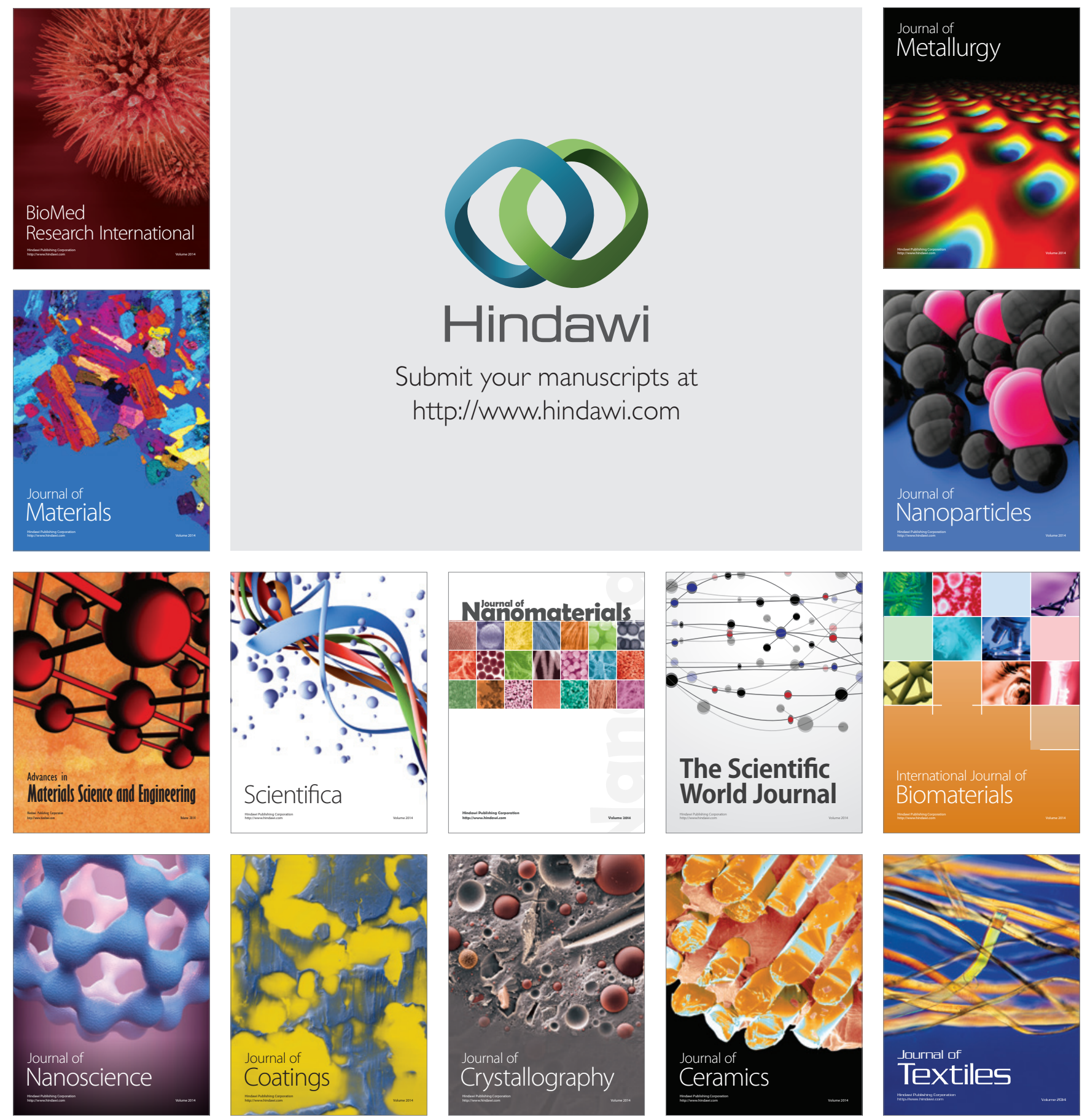